\section{Krebs im hohen Alter: Scores für eine gezielte Therapie}

$\mathrm{D}$ ie gesundheitliche Gesamtsituation älterer Menschen werde in der Krebstherapie oft nicht genug berücksichtigt, sagte Prof. Yon-Dschun Ko vom Johanniter-Krankenhaus Evangelische Kliniken Bonn. So sterben $40 \%$ der 70-Jährigen innerhalb von zehn Jahren an nicht onkologischen Erkrankungen. Die Wirksamkeit einer adjuvanten Therapie, durch die 2-4\% mehr Patienten zehn Jahre lang leben, werde allein schon deswegen statistisch ausgedünnt. Hinzu komme, dass infolge eingeschränkter Leber-, Nieren- und Hirnfunktion unerwünschte Wirkungen rascher eintreten und schwerer rückgängig zu machen sind. „Wir müssen uns deswegen bei alten Krebspatienten immer fragen, ob eine Therapie eigentlich noch relevant ist", so Ko. Entscheidend sei, die nicht krebsbe- zogene Gesamtprognose der Patienten zu bewerten.

Doch wie können diejenigen erkannt werden, die noch von einer Maximaltherapie profitieren? Imke Ortland, Klinische Pharmazie der Universität Bonn, plädierte für den Einsatz onkogeriatrischer Scores, wie den CRASH- und CARG-Score, die sowohl krebs- und krebstherapiebezogene als auch altersbezogene Faktoren einbeziehen und in Bonn derzeit klinisch evaluiert werden. In der Pilotphase mit 20 Krebspatienten jenseits der 70 bescheinigten die beiden Scores jeweils rund drei von vier Patienten ein mittleres bis hohes Risiko für starke unerwünschte Wirkungen. Die behandelnden Ärzte dagegen hielten 16 von 20 Patienten für fit genug für eine Maximaltherapie. Bei über 100 Patienten wird jetzt prospektiv überprüft, ob die Scores oder Ärzte die Patienten besser einschätzen.

Und wie kann eine sinnvolle Therapie im Alter so gestaltet werden, dass unerwünschte Wirkungen im Rahmen bleiben? Prof. Dr. Ulrich Jaehde, Pharmazeutisches Institut, Universität Bonn, stellte eine Studie vor, in der bei Krebspatienten in Abhängigkeit vom Risiko für unerwünschte Wirkungen wie Mucositis, Nausea/Emesis, Schmerzen oder Fatigue bestimmte Medikationsmanagementmodule durchlaufen wurden. Probleme sollten früh erkannt werden. Unabhängig vom Alter der Patienten ließen sich damit schwere unerwünschte Wirkungen im Mittel um einen Therapiezyklus verzögern, so Jaehde. Im nächsten Schritt soll das Bonner Medikationsmanagement um onkogeriatrische Assessments erweitert werden.

Dr. Philipp Grätzel von Grätz

"State of the Art: Medikationsmanagement beim älteren Krebspatienten", 26.2.2016

\title{
Mit Checkpoint-Hemmung gegen das Nierenzellkarzinom
}

$\mathrm{W}^{\mathrm{s}}$ ie beim Melanom gibt es bald auch bei Nierenkrebspatienten mit einem Checkpoint-Inhibitor eine neue Therapieoption. Durch Blockade des Rezeptors PD-1 auf aktivierten T-Lymphozyten verhindert Nivolumab die Wechselwirkung mit dem Liganden PD-L1 auf anderen Immunzellen und auf Tumorzellen. Der Effekt: Die ursprünglich ausgebremsten T-Zellen können die Tumorzellen attackieren. Die Zulassung von Nivolumab für Patienten mit einem Nierenzellkarzinom, die bereits onkologisch behandelt worden sind, wird noch im ersten Halbjahr 2016 erwartet. Grundlage der vorgeschlagenen Zulassungserweiterung sind die Ergebnisse der Phase-IIIStudie CheckMate 025 [NEJM 2015;373: 1803-13]. „Erstmals konnte in der Studie im Vergleich zur Therapie mit Everolimus ein signifikant verlängertes Gesamtüberleben gezeigt werden“, sagte Prof. Jens Bedke, Universitätsklinik Tübingen. Primärer Endpunkt der Studie mit mehr als 800 Patienten, die an einem fortgeschrittenen klarzelligen Nierenzellkarzi- nom erkrankt und bereits erfolglos antiangiogenetisch behandelt worden waren, sei das Gesamtüberleben gewesen. Median lebten die Patienten der Verumgruppe noch 25 Monate (95\%-Konfidenzintervall [KI] zwischen 21,8 und „noch nicht abschätzbar“), in der Vergleichsgruppe dagegen nur 19,6 Monate (95\%-KI: 17,6-23,1). Durch die Therapie mit dem PD-1-Hemmer wurde die Sterbewahrscheinlichkeit um $27 \%$ gesenkt (Hazard Ratio: 0,73; $\mathrm{p}=0,002$ ).

Darüber hinaus sprachen die Patienten der Nivolumab-Gruppe signifikant besser auf das Medikament an als die Patienten der Everolimus-Gruppe (objektive Ansprechrate: 25 vs. $5 \%$ ). Keinen statistisch signifikanten Unterschied gab es hingegen beim Parameter „progressionsfreies Überleben“. Geprüft wurde auch, ob der PD-1-Status einen Einfluss auf das Therapieergebnis hat. Nach Angaben von Bedke gab es jedoch keinen eindeutigen Zusammenhang zwischen Therapieantwort und der Stärke der Rezeptorexpression. Auffallend war das
Nebenwirkungsspektrum mit den modernen Therapieoptionen. „Es gibt eine neue Toxizitätsklasse“, so Bedke. Dazu gehören vor allem Fatigue, Durchfall und Entzündungen der Schleimhäute. In beiden Therapiegruppen klagte beispielsweise jeder Dritte über Fatigue. Künftig sei noch zu klären, ob es geeignete Biomarker für das Therapieansprechen beim Nierenzellkarzinom gibt.

Peter Leiner

Symposium „Immuntherapie des

metastasierten Nierenzellkarzinoms", 25.2.2016

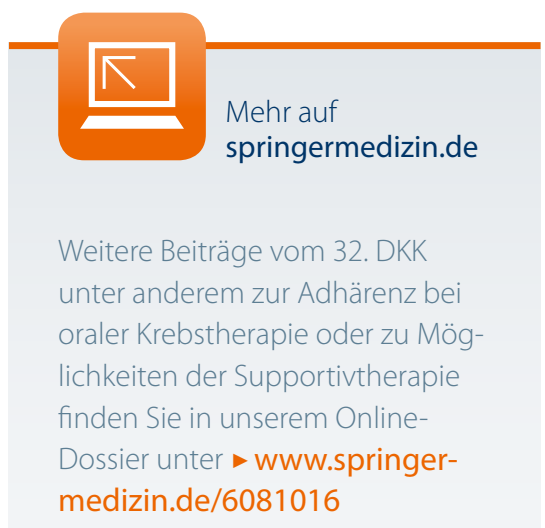

